\title{
Metastatic renal cancer and patient survival as a criterion of treatment response
}

\author{
GEORGE P. STATHOPOULOS, JOHN KOUTANTOS, SOTIRIS K. RIGATOS, \\ JOHN STATHOPOULOS, SPIROS BATZIOS and CHRISTINA BATZIOU
}

First Oncology Clinic, Errikos Dunant Hospital, Athens, Greece

Received July 25, 2007; Accepted October 15, 2007

\begin{abstract}
Advanced metastatic renal cancer is an incurable disease, unless a successful excision of metastatic lesions can be performed. No effective treatment has yet been found. In the last few years, targeting therapies have been developed. In the past, the main treatment was based on cytokines (interferon- $\alpha$ or interleukin-2). Our objective was to determine the median and overall survival in the 66 patients who were studied and reviewed. All had histologically confirmed advanced renal cancer. There were 41 male and 25 female patients, with a median age of 60 years. In $68.18 \%$ of the patients, the treatment was mainly interferon- $\alpha$ (IFN- $\alpha$ ) given 3 times a week for a median time duration of 6 months (range 3-12 months). Four patients received interleukin-2 (IL-2) and 17 patients received chemotherapy, 15 of whom had hormonal treatment. Eleven patients underwent palliative radiation therapy (in the bone or brain). Seven patients received no treatment apart from supportive care. A partial response was achieved in $11.11 \%$ of the patients treated with IFN- $\alpha$. No response was observed in patients treated with chemotherapy or hormonal therapy. The median survival of all the patients was 20 months (95\% CI 14.96-25.04). These results are discussed in comparison with the survival results of modern targeting treatment studies. In the latter studies, despite the high response rates (31-40\%), the survival was 16.4 months. Our data indicate that the response rate as a criterion is not adequate in determining drug effectiveness.
\end{abstract}

\section{Introduction}

Carcinoma of the kidney may be cured in a high percentage of patients by nephrectomy. Some patients are diagnosed in the advanced stage of the disease and others eventually develop metastases. Carcinoma of the kidney may be symptomless in the early stages, which can lead to a delayed diagnosis. Therefore, when the disease is advanced, radical surgical

Correspondence to: Dr G. P. Stathopoulos, Semitelou 2A, 11528 Athens, Greece

E-mail: dr-gps@ath.forthnet.gr

Key words: renal cancer, survival management may not be possible. Cytotoxic agents are only slightly effective (1) and no established chemotherapy schedule has been suggested. Biological response modifiers, cytokines, have been used and there are studies which describe results which are not of importance $(2,3)$. Interferon- $\alpha$ (IFN- $\alpha$ ) has been broadly used with some effectiveness; response rates vary from 6 to $15 \%(2,3)$. IFN- $\alpha$ also provided a $25 \%$ decrease in the risk of tumor progression and a modest survival benefit of 3-5 months when compared with a placebo equivalent $(3,4)$. Interleukin-2 (IL-2) has also been tested and several studies using a low or high dose of IL-2 showed responses ranging from 7 to $27 \%$ (5-7). Long-term responses have been observed (8). Toxicity may be intolerable, particularly when IL-2 is combined with IFN- $\alpha$ (4). The survival of certain patients may be longer without treatment, but this depends on the site of metastasis. A paramount effort has been made to search for new treatments which may inhibit the progression of the disease. A tyrosine kinase inhibitor, sunitinib, was introduced for the treatment of advanced renal cancer and has shown promising results in a Phase III trial (as described below). What should be the criterion of response for advanced renal cancer? The response rate, the duration of response, the time to tumor progression, the median survival or the overall survival? The present trial attempts to show whether the criterion of survival is a reliable one. The patients presented here were treated with chemotherapy or with cytokines, which may not have influenced survival. The study includes patient material from one hospital which was reviewed with the objective of determining whether the criterion of survival is the most important one.

\section{Materials and methods}

Patients with a histologically confirmed diagnosis of renal carcinoma were included. Based on clinical, radiological and CT-scan examinations, the patients had advanced or metastatic disease. Base-line examinations included clinical, full blood count, renal and liver function tests, assessment of functional status and ECOG performance status. Computed tomography (CT) of the chest and abdomen, magnetic resonance imaging (MRI) of the brain and cardiovascular examinations were also performed.

Treatment. Patients were treated either with chemotherapy or with IFN- $\alpha$ and very few with interleukin-2. Despite advanced 
Table I. Treatment.

\begin{tabular}{|c|c|c|c|}
\hline \multirow[b]{3}{*}{ Treatment } & \multicolumn{3}{|c|}{ Response } \\
\hline & \multirow[b]{2}{*}{$\mathrm{n}(\%)$} & PR & SD \\
\hline & & $\mathrm{n}(\%)$ & $\mathrm{n}(\%)$ \\
\hline INF- $\alpha$ & $45(68.18)$ & $5(11.11)$ & $20(44.44)$ \\
\hline IL-2 & $4(6.06)$ & - & 3 \\
\hline \multicolumn{4}{|l|}{ Chemotherapy } \\
\hline Vinblastine & $12(18.18)$ & - & $12(100)$ \\
\hline Vinblastine $+5-\mathrm{FU}$ & $2(3.03)$ & - & $2(100)$ \\
\hline Vinblastine + Bleomycin + Cisplatin (or anthracycline) & $3(4.54)$ & - & $3(100)$ \\
\hline \multicolumn{4}{|l|}{ Hormonal } \\
\hline Tamoxifen & $4(6.06)$ & - & $4(100)$ \\
\hline Medroxy-progesterone acetate & $11(16.66)$ & - & $11(100)$ \\
\hline \multicolumn{4}{|l|}{ Radiation therapy } \\
\hline Skeleton & $8(12.12)$ & - & $\mathrm{NR}^{\mathrm{a}}$ \\
\hline Brain & $3(4.54)$ & - & NR \\
\hline Untreated & $7(10.60)$ & - & - \\
\hline
\end{tabular}

${ }^{\mathrm{a} N R}$, no response.

disease, a percentage of patients remained in a good condition with a good performance status which permitted long survival without any specific treatment (chemotherapy or biological response modifiers), with or without supportive treatment. A follow-up and clinico-laboratory testing was performed monthly. IFN- $\alpha$ (5 million units per day, 3 times a week) was administered to 48 patients and was planned to be given for 1 year. Three patients refused to continue treatment after the first 3 or 4 doses due to the side effects such as malaise and high fever. The 45 patients who continued IFN- $\alpha$ had a median duration of treatment of 6 months (range 3-12 months); 3 of these patients had repeated IFN- $\alpha$ after the first year for another period of 6-12 months. IL-2 (18 million units daily for 5 days) was given to 4 patients, one of whom received it in combination with IFN- $\alpha$ and to the other 3 as a single treatment for 5 days every four weeks. None of these patients had more than 2 courses, since toxicity was intolerable. Chemotherapy was administered to 17 patients, 7 of whom had chemotherapy after IFN- $\alpha$ plus hormonal treatment with either tamoxifen of medroxy-progesterone acetate. Another 10 patients were treated with chemotherapy or/and hormonotherapy. The cytotoxic agent given was mainly vinblastine and additional agents were bleomycin, cisplatin, or anthracycline. Treatment is shown in Table I. Seven patients were given no treatment and only had supportive care. Eleven patients had radiation therapy, 8 for bone metastases and 3 for brain metastases.

Definition of response. A complete response (CR) was defined as the disappearance of all measurable or evaluable disease, signs and symptoms and biochemical changes related to the tumor for at least 4 weeks during which time no new lesions may appear; partial response (PR), a $>30 \%$ decrease in tumor burden, also confirmed at 4 weeks at the earliest; in stable disease (SD) neither PR nor progressive disease (PD) criteria were met; PD, a $20 \%$ increase in tumor burden and no CR, PR, or SD before increased disease. Response data were based on the Response Evaluation Criteria in Solid Tumors (RECIST) (9). A two-step deterioration in performance status, a $>10 \%$ loss in pretreatment weight or increasing symptoms did not by themselves constitute progression of the disease; however, the appearance of these complaints was followed by a new evaluation of the extent of the disease. All responses had to be maintained for at least 4 weeks and had to be confirmed by two independent radiologists and two experienced oncologists.

Statistical design. The primary end-point of this study review was to determine overall survival and tolerance. Overall survival was calculated from the day of enrollment until death, or the end of the study. The median probability of survival and the median time to tumor progression (TTP) were estimated by the Kaplan-Meier method.

\section{Results}

From 1997-2007, 66 patients were enrolled in this study review. The patients' characteristics are shown in Table II. There were 41 male and 25 female patients (median age of 60 years, range 30-76 years). The majority of patients had a performance status of 0 and 1 (ECOG scale).

Response. The majority $(49 / 66,74.24 \%)$ of the patients were treated with biological response modifiers. Forty-five patients received IFN- $\alpha$ and 4 had IL-2. Five (11.11\%) patients who received INF- $\alpha$ treatment achieved a partial response of at least 3 months duration. No other responses were observed in any of the remaining treatments that were administered. Stable disease of long duration was common in all other treatments. 
Table II. Patients' demographics and characteristics.

\begin{tabular}{|c|c|c|}
\hline & No. & $\%$ \\
\hline Patients enrolled & 66 & 100 \\
\hline \multicolumn{3}{|l|}{ Gender } \\
\hline Male & 41 & 62.12 \\
\hline Female & 25 & 37.88 \\
\hline \multicolumn{3}{|l|}{ Age (yr) } \\
\hline Median & 60 & \\
\hline Range & $30-76$ & \\
\hline \multicolumn{3}{|l|}{ Performance status (ECOG) } \\
\hline 0 & 25 & 37.88 \\
\hline 1 & 32 & 48.48 \\
\hline 2 & 9 & 13.63 \\
\hline Primary disease site: renal & 66 & 100 \\
\hline \multicolumn{3}{|c|}{ Histology: adenocarcinoma; site of metastasis } \\
\hline Lung & 27 & 40.91 \\
\hline Abdomen & 20 & 30.30 \\
\hline Bone & 7 & 10.61 \\
\hline Liver & 4 & 6.06 \\
\hline Brain and lung & 8 & 12.12 \\
\hline Total & 66 & \\
\hline
\end{tabular}

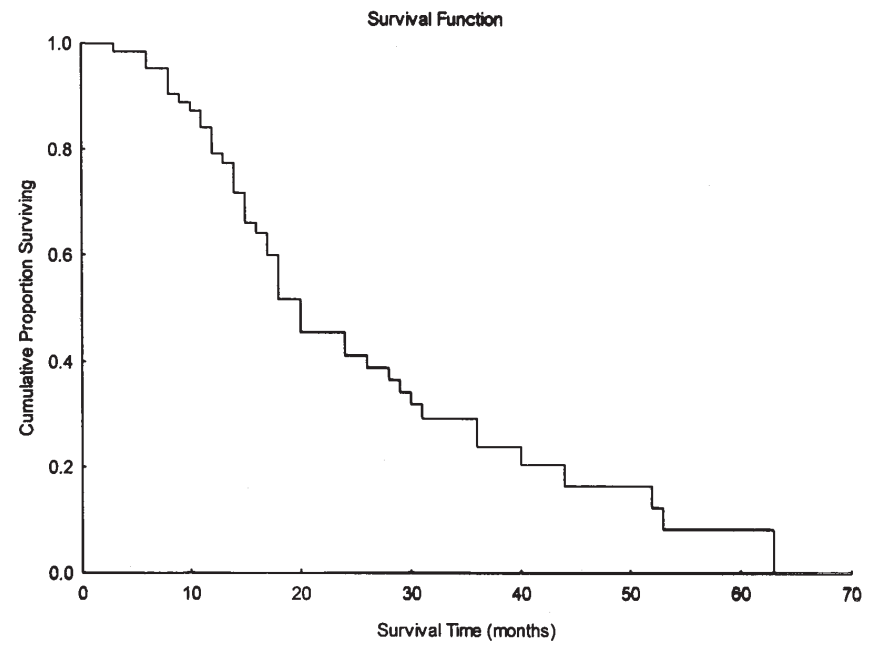

Figure 1. Kaplan-Meier survival curve.

Radiation therapy was mainly applied to patients with bone metastases, for relief. Radiotherapy was also administered in patients with brain metastases, without effectiveness.

Toxicity. No serious adverse reactions were seen during the course of the treatment. Only 3 patients stopped IFN- $\alpha$, due to toxicity, as did 4 patients that were treated with 2 courses of IL-2. The patients treated with chemotherapy had minor myelotoxicity and asthenia. Patients who had been on a longduration (3-12 months) of IFN- $\alpha$, had a high fever and malaise after the first or second injection. During the rest of the duration of IFN- $\alpha$ treatment, nearly all patients experienced low fever and mild malaise. In general, the treatment was well-tolerated.

Survival data. The median follow-up was 4 years (range 210 years). The median survival time was 20.0 months and the mean survival time, 26.5 months (95\% CI 14.9 -25.04 and $21.5-31.4$, respectively). Fig. 1 shows the Kaplan-Meier survival curve.

\section{Discussion}

Advanced stage renal cancer is an incurable disease unless there are metastatic lesions which can be excised by surgery (10); this does occur in a small percentage of patients but it is unfortunate that no effective treatment exists. Patients may have a long survival which is mainly dictated by the slowgrowing property of the disease. The management of a patient with advanced metastatic renal cancer requires certain criteria on which to base the effectiveness of the agent given. The main criteria used in any malignancy for an agent's efficacy are the response rate and median and overall survival. The response rate observed after treatment may be of no value if it is not accompanied by survival prolongation. In slow-growing malignant diseases such as breast cancer, colorectal cancer, renal cancer and some types of soft tissue sarcomas, the criterion of survival time needs very careful consideration. With these diseases, only randomized trials may give a reliable outcome with respect to survival. The patients in the experimental study arm should be very well balanced with the patients in the control arm. It is worth looking at studies using 
different treatments from the perspective of response and survival. Chemotherapy is not considered effective $(11,12)$ as the response rate is $>10 \%$, with the exception of gemcitabine with 5-fluorouracil, which rendered a $17 \%$ response rate (13). Renal cell cancer is also considered to be resistant to radiation which has only a palliative effect in bone metastases (14). Biological therapy has been shown to be more effective than chemotherapy in advanced renal cell carcinoma.

A trial that compared INF- $\alpha$ with vinblastine showed a survival benefit of 7.5 months for the former (15). A survival benefit of 2.5 months for INF- $\alpha$ was observed when compared with megestrol (2). Responses with INF- $\alpha$ varied from 5 to $15 \%$ and the duration of response was limited to 4-6 months (16). IL-2 which activates T cells and natural killer cells, has been shown in high doses to produce a $20 \%$ response rate in renal cell carcinoma. It has also been reported that $7 \%$ of the responders achieved a complete response $(17,18)$. The high toxicity, occasionally intolerable, is an inhibitory factor for the use of IL-2. Attempts to reduce the toxicity have been made by decreasing the dose of IL-2, and although lower toxicity was achieved, responses were also decreased $(8,19)$. New agents, classified as targeted biologic therapy, have recently been developed. Temsirolimus (rapamacin) is one which has shown activity. Sorafenib, a broad spectrum kinase inhibitor, is another which also seems to have an impressive response rate; however, the patients who had $>50 \%$ tumor shrinkage and those that had $25 \%$, showed no statistically significant difference with regard to progression-free survival (PFS). It was pointed out that on the basis of these results, classic response criteria are not the best measure of response (20,21).

A Phase III trial using sorafenib in 503 patients refractory to cytokine therapy, reported a prolongation of PFS to 24 weeks compared to the 12 weeks of the placebo group (22). Another targeting agent, sunitinib (Sutent), also a multitargeted inhibitor, has shown promising results. In a recent trial in renal cell carcinoma, the partial response that was given was $40 \%$ and an additional $27 \%$ stable disease was observed. The median time to disease progression was 8.7 months and the median survival was 16.4 months (23). The same agent was used in a multicenter randomized trial of 750 patients with metastatic renal cell carcinoma and was compared with INF- $\alpha$ with respect to PFS and response rate (24). It was found that PFS was significantly longer (11 months) than that of INF- $\alpha$ (5-months). The response rate was higher with sunitinib (31\%) versus that of INF- $\alpha(6 \%)$. No significant difference was found in the interim analysis in the median survival between the two groups; at the end of their study, the final survival analysis had not yet been done. These studies have reported a median survival that is not higher than 16.4 months.

In our present trial where the main treatment was INF- $\alpha$ in $68.18 \%$ of our patients, the median survival was 20 months, and $20 \%$ of the patients survived for over 3 years; half of the patients remained without treatment for a long period of time. The choice of PFS as a criterion of a treatment's efficacy in the aforementioned studies using targeting agents, appears to be inadequate. In an incurable disease, the importance of the overall survival and quality of life are priorities. Our studyreview showed a higher median survival than that shown in the trials with sorafenib and sunitinib, despite the very low response $(11 \%)$ of INF- $\alpha$ in our study versus the very high 31 and $40 \%$ observed with sunitinib. There may be an explanation for this discrepancy. Those patients with advanced metastatic renal cancer may have a long survival due to the slow-growing disease. This explains why, whatever the treatment given, be it chemotherapy, radiation, biological response modifiers and recent targeting therapies, the criteria of response and PFS appear to be of little importance in documenting the effectiveness of the treatment.

In conclusion, in advanced renal cancer, the response rate should not be considered as a reliable criterion for treatment effectiveness. This disease can be classified as 'dormant' (i.e. slow growing) and only survival should be the criterion eligible for treatment effectiveness.

\section{References}

1. Stadler WM, Huo D, George C, et al: Prognostic factors for survival with gemcitabine plus 5-fluorouracil based regimens for metastatic renal cancer. J Urol 170: 1141-1145, 2003.

2. Median Research Council Renal Cancer Collaborators: Interferon-alpha and survival in metastatic renal carcinoma. Early results of a randomized central trial. Medical Research Council Renal Cancer Collaborators. Lancet 353: 14-17, 1999.

3. Motzer RJ, Bacik J, Murphy BA, et al: Interferon-alpha as a comparative treatment for clinical trials of new therapies against advanced renal carcinoma. J Clin Oncol 20: 289-296, 2002.

4. Coppin C, Porzsolt F, Awa A, et al: Immunotherapy for advanced renal cell cancer. Cochrane Database Syst Rev (1): CD 001425, 2005.

5. Rosenberg SA, Lotze MT, Yang JC, et al: Prospective randomized trial of high-dose interleukin-2 alone or in conjunction with lymphokine-activated killer cells for the treatment of patients with advanced cancer. J Natl Cancer Inst 21: 622-632, 1993.

6. Fyfe G, Fisher RI, Rosenberg SA, et al: Results of treatment of 255 patients with metastatic renal cell carcinoma who received high-dose recombinant interleukin-2 therapy. J Clin Oncol 13: 688-696, 1995.

7. McDermott DF, Regan MM, Clark JI, et al: Randomized phase III trial of high-dose interleukin-2 versus subcutaneous interleukin-2 and interferon in patients with metastatic renal cell carcinoma. J Clin Oncol 23: 133-141, 2005

8. Yang JC, Sherry RM, Stemberg SM, et al: Randomized study of high-dose and low-dose interleukin- 2 in patients with metastatic renal cancer. J Clin Oncol 21: 3127-3132, 2003.

9. Therasse P, Arbuck SG, Eisenhower EA, et al: New guidelines to evaluate the response to treatment in solid tumors. J Natl Cancer Inst 92: 205-216, 2000.

10. Pfannschmidt J, Hoffmann H, Muley T, et al: Prognostic factor for survival after pulmonary resection of metastatic renal cell carcinoma. Ann Thorac Surg 74: 1653-1657, 2002.

11. Amato RJ: Chemotherapy for renal cell carcinomas. Semin Oncol 27: 177-186, 2000.

12. Lilleby W and Fossa SD: Chemotherapy in metastatic renal cell cancer. World J Urol 23: 175-179, 2005.

13. Rini BI, Vogelzang NJ, Dumas MC, et al: Phase II trial of weekly intravenous gemcitabine with continuous infusion fluorouracil in patients with metastatic renal cell cancer. J Clin Oncol 18: 2419-2426, 2000.

14. Dibiase SJ, Valicenti RK, Schultz D, et al: Palliative irradiation for focally symptomatic metastatic renal cell carcinoma: support for dose escalation based on a biological model. J Urol 158: 746-749, 1997.

15. Pyrhomen S, Salminen E, Ruutu M, et al: Prospective randomized trial of interferon alpha-2a plus vinblastine versus vinblastine alone in patients with advanced renal cell cancer. J Clin Oncol 17: 2859-2867, 1999.

16. Fossa SD: Interferon in metastatic renal cell carcinoma. Semin Oncol 27: 187-193, 2000.

17. Rosenberg SA, Yang JC, Topalian SL, et al: Treatment of 283 consecutive patients with metastatic melanoma or renal cell cancer using high-dose-bolus interleukin 2. JAMA 271: 907-913, 1994. 
18. Fyfe GA, Fisher RI, Rosenberg SA and Parkinson DR: Response data for 255 patients with metastatic renal cell carcinoma treated with a high-dose recombinant interleukin-2 therapy. J Clin Oncol 14: 2410-2411, 1996.

19. Negrier S, Escudier B, Lasset C, et al: Recombinant human interleukin-2 recombinant human interferon alpha-2a or both in metastatic renal-cell carcinoma, Groupe Français d'Immunothérapie. N Engl J Med 338: 1272-1278, 1998.

20. Ratain MJ, Eisen T, Stadler WM, et al: Phase II placebo controlled randomized discontinuation trial of sorafenib in patients with metastatic renal cell carcinoma. J Clin Oncol 24: 2505-2512, 2006.

21. Nelson EC, Enavs CP and Lara Jr PN: Renal cell carcinoma: Current status and emerging therapies. Cancer Treat Rev (In press).
22. Eisen T, Bukowski RM, Staehler M, et al: Randomized phase III trial of sorafenib in advanced renal cell carcinoma (RCC): impact of crossover on survival. J Clin Oncol 245 (Abs. 4524), 2006.

23. Motzer RJ, Michaelson DM, Redman BC, et al: Activity of SU11248, a multitargeted inhibitor of vascular endothelial growth factor receptor and platelet-derived growth factor receptor in patients with metastatic renal cell carcinoma. J Clin Oncol 24: 16-24, 2006.

24. Motzer RJ, Hutson TE, Pharm DO, et al: Sunitinib versus interferon alpha in metastatic renal-cell carcinoma. $\mathrm{N}$ Engl $\mathrm{J}$ Med 356: 115-124, 2007. 